\title{
The person behind the nodule: a narrative review of the psychological impact of lung cancer screening
}

\author{
Samantha L. Quaife ${ }^{1}$, Samuel M. Janes ${ }^{2}$, Kate E. Brain ${ }^{3}$ \\ ${ }^{1}$ Centre for Cancer Prevention, Wolfson Institute of Preventive Medicine, Barts and The London School of Medicine and Dentistry, Queen Mary \\ University of London, London, UK; ${ }^{2}$ Lungs for Living Research Centre, UCL Respiratory, Division of Medicine, University College London, \\ London, UK; ${ }^{3}$ Division of Population Medicine, School of Medicine, Cardiff University, Cardiff, UK \\ Contributions: (I) Conception and design: All authors; (II) Administrative support: None; (III) Provision of study materials or patients: None; (IV) \\ Collection and assembly of data: None; (V) Data analysis and interpretation: All authors; (VI) Manuscript writing: All authors; (VII) Final approval of \\ manuscript: All authors. \\ Correspondence to: Samantha L. Quaife. Centre for Cancer Prevention, Wolfson Institute of Preventive Medicine, Barts and The London School of \\ Medicine and Dentistry, Queen Mary University of London, London, UK. Email: s.quaife@qmul.ac.uk.
}

\begin{abstract}
Lung cancer is the leading cause of cancer mortality globally, responsible for an estimated 1.76 million deaths worldwide in 2018 alone. Screening adults at high risk of lung cancer using low-dose computed tomography (LDCT) significantly reduces lung cancer mortality by finding the disease at an early, treatable stage. Many countries are actively considering whether to implement screening for their highrisk populations in light of the recently published Dutch-Belgian trial 'NELSON'. In deciding whether to implement a national screening programme, policymakers must weigh up the evidence for the relative risks posed to the entire screened population, including the potential psychological burden. This narrative review aimed to critically summarise the evidence for both negative and positive psychological responses experienced throughout the LDCT screening pathway, to describe their magnitude, duration and clinical relevance, and to draw out different aspects of measurement design crucial to their interpretation. A further aim was to discuss the available evidence for individual differences in psychological response, as well as interventions designed to promote psychological well-being. In summary, there was no evidence that the LDCT screening process caused adverse psychological outcomes overall, although those receiving indeterminate and suspicious LDCT results did report clinically raised anxiety and lung cancer-specific distress in the shortterm. There was early evidence that demographic factors, smoking status and screening-ineligibility could be associated with individual differences in propensity to experience distress. Qualitative data suggested health beliefs could be modifiable mediators of these individual differences, but their aetiology requires quantitative and prospective research. There was also some evidence of positive psychological responses that could be capitalised on, and of the potential for person-centred communication interventions to achieve this. Further research needs to be embedded in real-world LDCT lung cancer screening services and use conditionspecific measures to monitor outcomes and test evidence-based communication interventions in promoting psychological well-being.
\end{abstract}

Keywords: Anxiety; depression; early detection of cancer; lung neoplasms; quality of life

Submitted Nov 09, 2020. Accepted for publication Mar 12, 2021.

doi: $10.21037 /$ tlcr-20-1179

View this article at: http://dx.doi.org/10.21037/tlcr-20-1179 


\section{Introduction}

Decades of clinical trials sought to establish an effective screening test for lung cancer; a disease that can be treated curatively when diagnosed early yet claimed over 1.7 million lives globally in 2018 alone (1). The US National Lung Screening Trial's (NLST) findings were therefore groundbreaking; showing for the first time, a $20 \%$ relative risk reduction in lung cancer mortality from screening highrisk adults (due to age and smoking history) annually using low-dose computed tomography (LDCT) compared with chest X-ray (2). This led to its recommendation by several medical bodies in the US, including the US Preventive Services Task Force (USPSTF) (3). Countries elsewhere awaited data from the Dutch-Belgian trial 'NELSON' which recently published a $24 \%$ relative risk reduction for high-risk men (4) spurring renewed calls for implementation.

Reduction in mortality is of course not sufficient to warrant the implementation of a population-based screening programme; a benefit that relatively few participants in screening programmes will experience personally. In addition to broader criteria for implementation [such as acceptability, quality assurance, resource, equity $(5,6)]$, policymakers must weigh up evidence for the relative risks posed to the entire screened population. These include the possible psychological costs of participating in screening; most obviously the potential for abnormal screening results and follow-up to cause undue distress. This may be especially important to understand in the context of lung cancer screening because a significant proportion of individuals experience 'indeterminate' pulmonary nodules which are unlikely to be cancer but require surveillance. Two systematic reviews of patient-centred outcomes in 2014 (7) and 2016 (8) found only short-term psychological distress following abnormal screening results but concluded further high quality research is needed, including 'real world' implementation. Since these reviews were published, further trials in different countries have published their psychological outcome data, including a real-world demonstration design. More recent research has also begun to suggest a broader myriad of diverse psychological consequences than those previously reviewed which occur throughout the screening pathway, not just following disclosure of results, and which may include positive effects $(9,10)$.

Any psychological risks or benefits are also important to understand from the individual perspective, to support autonomous informed decision-making and psychological preparedness, as well as from a system-level perspective, to ensure person-centred services are designed to mitigate potential risk, optimise well-being and capitalise on opportunities for adaptive psychological outcomes and behaviour. The current narrative review aimed to critically summarise state-of-the-art evidence for the psychological impact of LDCT lung cancer screening to-date. The specific objective was to examine the different types of psychological response that have been investigated in terms of their magnitude, duration and clinical relevance, as well as the factors affecting an individual's propensity to experience these psychological responses. A secondary objective was to summarise any available evidence for how lung cancer screening services should be designed to promote psychological well-being. We present the following article in accordance with the narrative review reporting checklist (available at http://dx.doi.org/10.21037/ tlcr-20-1179).

\section{Methods}

A rapid search of two electronic databases (PubMed and PsychInfo) was carried out to identify original research studies of quality of life and psychological outcomes from LDCT lung cancer screening of high-risk adults that had been published in English language on any date up until $30^{\text {th }}$ June 2020 in international peer-reviewed journals. All types of study design were eligible for inclusion. Due to a lack of research examining interventions for improving psychological outcomes from LDCT screening, the search was expanded to include original research studies of personcentred communication in the incidental pulmonary nodule context.

\section{Narrative discussion}

\section{Types of psychological outcome}

Studies investigating the psychological impact of LDCT lung cancer screening have predominantly focussed on understanding whether the process causes clinically significant psychological morbidity, especially among those receiving abnormal results. However, heterogeneity in the measures used, as well as their timing and sensitivity (i.e., generic- or condition-specific) are critical to interpreting these findings. Therefore, the following sections present the available evidence by the type of outcome measured, with specific reference to these measurement aspects of study design. This is supported by Table 1 which presents 







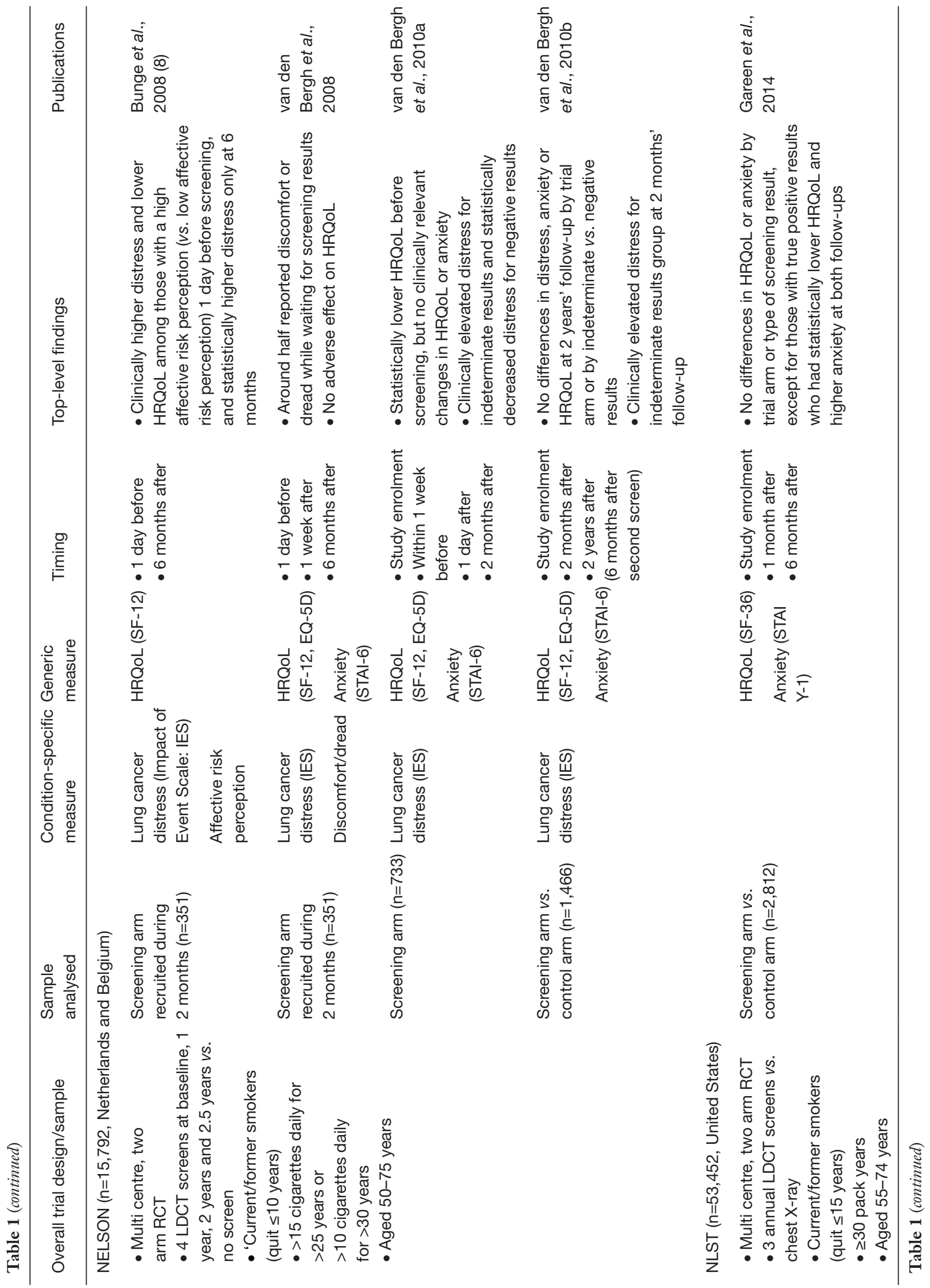




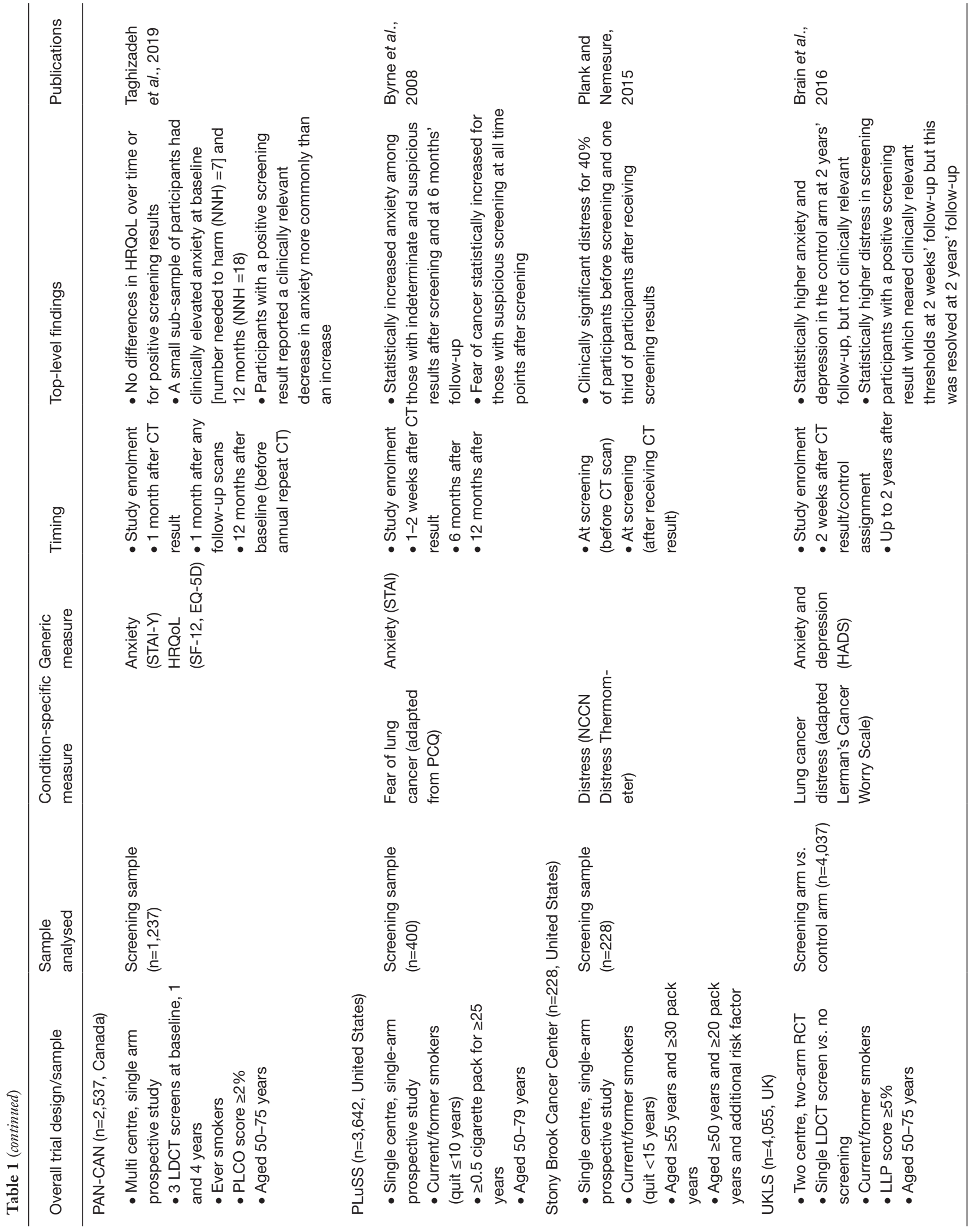


an overview of the design and findings of psychological outcome studies in LDCT screening trials.

\section{Health-related quality of life (HRQoL)}

Three trials of LDCT lung cancer screening have published the HRQoL outcomes of their participants with all using either the 12 or 36 item version of the Short Form Health Survey (SF-12/SF-36) and the EuroQol questionnaire (EQ-5D). These are generic measures of HRQoL which assess global aspects of both physical and mental functioning and are widely used because they allow comparison across different disease and healthy populations using standardised scores. In doing so, however, they are less sensitive to those aspects of HRQoL that are specific to a particular disease or patient group and could miss important consequences from the individual's perspective. Nevertheless, using these measures, the NLST (11), NELSON (12-14) and Pan-Canadian Early Detection of Lung Cancer Study (PAN-CAN) trials (15) found no evidence of any clinically adverse impact of LDCT lung cancer screening overall on HRQoL across their respective immediate (1 day), short-term (1 and 2 months) and long-term (6 months, 1 year, 2 years) follow-up points. The same was true when comparing HRQoL between the different LDCT result sub-groups, including indeterminate results received at both prevalence and incidence screening rounds (14), although with the exception of those receiving true positive results (11). It is important to note here that the NLST did not distinguish indeterminate results from those which were immediately suspicious for lung cancer. Nevertheless, the consistency of these findings over time, internationally, and with large, statistically powered samples suggests they are likely to be reliable. However, further monitoring within real world screening services is warranted because the 'healthy volunteer' bias observed in trial participation may mean those most likely to experience decline, in physical or mental functioning have been underrepresented to-date (16-19).

\section{Psychological morbidity: depression and anxiety}

While generic HRQoL measures include dimensions of psychological and mental functioning, some studies have used measures of clinical psychological morbidity, either instead of or in combination with HRQoL, in order to focus on the psychological consequences of screening specifically. The UK Lung Screening Trial (UKLS) and the
Lung Screen Uptake Trial (LSUT) both used the Hospital Anxiety and Depression Scale (HADS) following a single, one-off screening episode. Neither found clinically relevant differences in anxiety or depression at the one day (LSUT), two weeks (UKLS), 3 months (LSUT) or 2 years (UKLS) follow-up points for their screened cohorts overall. This was the case when compared with UKLS's non-screen control arm (20) and LSUT's 'screening unaware' community comparison sample who had the same age and smoking characteristics as the screening cohort, but were unaware of screening (21). In fact, it was UKLS's control arm and those in LSUT's screening cohort who were ineligible for screening who reported statistically significantly higher anxiety and depression scores, when compared with the intervention arm and community comparison sample, respectively.

The trends in anxiety and depression scores among participants receiving abnormal LDCT results (i.e., indeterminate or incidental) were similar to those observed for HRQoL. Statistically significantly higher anxiety scores were observed at short-term follow-up, but scores remained within the 'normal' clinical range and longer-term followup by UKLS ( 2 years) found any increases in anxiety had diminished. Participants with a suspicious thoracic lesion were more likely to report clinically significant moderate or severe depression scores at 3 months' follow-up within LSUT (21). The extension of findings from UKLS's trial setting to LSUT's 'real world' demonstration service design, supports their external validity and generalisability to high risk, previously underrepresented groups. However, the relatively small number of cases within the abnormal LDCT result sub-groups in LSUT means these findings should be treated cautiously. Future research should aim to embed psychological morbidity measures within real-world services to achieve an ecologically valid and statistically powered assessment for abnormal LDCT result sub-groups.

It could also be argued that distress induced by abnormal results during a one-off screening episode would be expected to have a shorter duration than within a repeat screening programme, because the 'screenee' no longer has the prospect of further screening rounds which could have a cumulative impact. However, a similar pattern of anxiety has been observed among participants of trial and cohort studies undergoing repeat screening intervals using the State Trait Anxiety Inventory (STAI); a measure of transitory anxiety in that moment ('state') and as a stable personal characteristic ('trait'). The NELSON, NLST, PAN-CAN and Pittsburgh Lung Screening Study (PLuSS) found no clinically relevant 
changes in STAI scores for their screening samples overall across differing short-term (before screening, next day, post-result, 1 month, 2 months) and long-term (6 months, 1 year, 2 years) time points $(11,13,15,22)$. This was also true when stratified by type of LDCT result, with the exception of those in the true positive group, and for the PLuSS study, those with indeterminate results directly after receiving their results (22). State anxiety in this indeterminate group remained elevated at 6 months' followup but decreased over 12 months. In contrast, those in the PAN-CAN study who received a positive screening result more frequently reported a reduction in state anxiety than an increase (15). Taghizadeh and colleagues suggest their personalised method of communicating results could have played a role in reducing anxiety among this group but warn against overinterpreting this finding, given the relatively small absolute number of cases reporting a reduction in anxiety. Furthermore, their 'positive screen' group included both indeterminate findings as well as those needing investigations for lung lesions. Future research should test the effectiveness and feasibility of personalised methods of results communication in improving psychological outcomes, and where possible, analyse the outcomes of different abnormal LDCT result groups separately.

\section{Specific measures of psychological distress}

Some trials have also included condition-specific or eventspecific measures of distress, because they may be more sensitive to lung cancer screening-induced distress than generic measures of anxiety, depression and HRQoL. For example, while the Danish Lung Cancer Screening Trial (DLCST) found no increase in prescribed anxiolytic or antidepressant medication for their intervention arm over 3 years' follow-up (23), their condition-specific measure of the psychological consequences of lung cancer screening [COS-LC; (24)] did show statistically significant negative psychosocial responses for behaviour (e.g., concentration, withdrawal), dejection (e.g., feeling sad or uneasy) and sleep (e.g., difficulty falling asleep, waking up early) over five annual screening rounds (25). However, it was those in the control arm of the trial (who did not undergo LDCT screening) who reported the most negative psychosocial consequences when compared with the intervention arm across the final 3 years of the five annual screening rounds (25). This is similar to the statistically (but not clinically) higher anxiety and depression scores observed among UKLS's control arm (20), and LSUT's screening- ineligible participants (21). Together, these findings suggest that the availability of lung cancer screening could cause psychological distress among individuals who feel at high risk of lung cancer, either subjectively (one's own risk perception) or objectively (being eligible for a lung screening trial), if screening is subsequently denied. They also raise the possibility that screening for lung cancer could lower distress among its participants, perhaps by providing a means of proactively managing risk of lung cancer mortality and related concern. Research should seek to inform the design of careful, evidence-based communication about eligibility and ineligibility at both the population and individual level which promotes positive psychological and behavioural outcomes.

With regards to the different LDCT screening results, only one study has reported any impact on those receiving negative results (referred to hereon as 'normal results' to avoid confusion with negative psychological effects). In the NELSON trial, these participants reported a significant decrease in intrusive thoughts [as measured by the Impact of Event Scale (26)] at 2 months' follow-up suggesting a positive psychological effect for this group in the shortterm. Instead, studies have more frequently observed that abnormal results induce distress about lung cancer in the immediate and short-term although that this, like with more generic anxiety measures, resolves over time. For example. those receiving positive results (both true and false) within the DLCST reported negative psychosocial effects at one week and 1 month, but these were not sustained at six and 18 months' follow-up (27). Trials have however differed in their conclusions about the clinical relevance of their findings. The NELSON trial found intrusive thoughts about cancer were clinically significantly raised at 2 months' follow-up among those with indeterminate results when compared with their baseline scores, but resolved at 2 years' follow-up (14). The UKLS and LSUT, which used adapted versions of Lerman's Cancer Worry Scale (28), also found raised lung cancer distress among those in the indeterminate as well as the urgent referral results group at their 2 and 3 months' follow-up points, respectively. However, unlike the NELSON trial, both found that the absolute differences were not clinically relevant. For example, in UKLS, cancer distress remained within clinically 'normal' ranges for those in the indeterminate group, although did near clinically relevant thresholds for those receiving urgent referrals for cancer at 2 months' follow-up (20,21). Conversely, the PLuSS study, found no change in lung cancer specificdistress among those with indeterminate results, either 
clinically or statistically, when using a measure of lung cancer fear adapted from the Consequences of Screening Questionnaire (22). Fear of cancer was significantly increased among those with suspicious results and remained so at 12 months' follow-up.

There is some evidence that the screening appointment itself and time spent waiting for results may be sources of lung cancer-specific distress. The Stony Brook Cancer Center found that $43 \%$ of participants in their cohort study $(n=228)$ experienced raised lung cancer-specific distress immediately before having screening (29), while LSUT found statistically significantly higher reported lung cancer-specific distress at the screening appointment when compared with a matched community comparison sample who were unaware of screening, but the absolute difference in mean scores was small (21). In NELSON, participants reported a statistically significant increase in intrusive thoughts about lung cancer when waiting for their screening results, with half reporting dread or discomfort in a smaller sub-study $(\mathrm{n}=351)(13)$. However, at the same time, NELSON participants reported a decline in generic anxiety scores. This suggests that the anticipation of screening results could trigger specific distress about the chances of having lung cancer among some, but that this does not cause more generalised symptoms of anxiety. Nevertheless, it is important to ensure screening results are shared with participants as soon as is practically possible, and that the expected waiting period for results is clearly communicated.

\section{Diverse positive and negative psychological responses}

Research has begun to identify diverse cognitive and affective responses to LDCT screening, which move beyond psychological morbidity and distress, to include positive as well as negative dimensions. Qualitative interviews carried out with LSUT participants identified a broad spectrum of responses experienced at every stage of the screening pathway that varied both between different, and within the same, individuals (9). These included, for example, perceived risk of lung cancer (higher, unchanged or lower), perceived control over respiratory health (empowered or fatalistic), focus on cancer (relieved or preoccupied), reassurance (temporary and appropriate or overly reassured) and lung cancer symptom vigilance (attentive to previously unacknowledged symptoms). These more nuanced responses are important to understand because they may mediate individual differences in psychological well-being following screening and provide modifiable factors for promoting positive psychological outcomes. They might also have important downstream consequences for future early diagnosis and prevention behaviour, including timely symptomatic presentation, adherence to future screening rounds, and smoking abstinence (9).

The most studied of these responses to date is screening participants' perceptions of their risk of lung cancer; particularly among those who remain dependent on tobacco smoking. Harris (2015) warned of the potential 'cognitive harm' screening could cause to some heavy smokers, following qualitative research (30) in which half of the veterans interviewed reported at least one misperception following screening that could undermine motivation to stop smoking. These included the belief that CT screening confers the same benefit as smoking cessation and that a normal screening result means the individual will not suffer harms of smoking. However, there is no quantitative evidence from the trial context that screening causes those who smoke or those who receive a normal LDCT screen to underestimate their risk of lung cancer. In the NLST, which measured both personal and comparative risk perceptions, participants' risk perceptions for lung cancer and other smoking-related disease were unchanged at 1 years' follow-up when compared with those they reported prior to screening (31). This was true across the different LDCT screening result sub-groups, including those who had a normal screen. More generally, it appeared to be former smokers who were the most likely to underestimate their risk of lung cancer, with current smokers who had the highest cigarette consumption reporting the highest risk perceptions (32,33). In PLuSS, while participants' perceived risk of lung cancer did decrease following a normal result, they remained higher than their objective risk regardless of the type of LDCT result they received (22). Future research could use a prospective, longitudinal design to understand how individuals' risk perceptions might change across time in response to multiple screening rounds and the accumulation of different types of LDCT results.

\section{Individual differences in psychological response}

While abnormal LDCT results have been an understandable focus of psychological studies, research has begun to implicate sociodemographic factors, smoking history and health beliefs as potential risk factors for explaining individual differences in screening-induced distress. 


\section{Sociodemographic characteristics}

In UKLS, LSUT, PLuSS and the Stony Brook Cancer Center study, women reported statistically, but not clinically, higher cancer distress and anxiety scores than men $(20-22,29)$, and in NELSON and PAN-CAN, lower HRQoL $(14,15)$. This trend was observed over time and across both trial arms (screening or no screening) in UKLS, and persisted at 2 years' follow-up. It was also observed at baseline for LSUT and Stony Brook Cancer Center participants, but not at LSUT's 3 months' follow-up after adjusting for baseline lung cancer worry scores. It is possible that enrolment into a screening trial or programme, which prompts conscious comprehension of lung cancer risk, is relatively more anxiety-provoking for women than men. Alternatively, perhaps women who are worried about lung cancer are more motivated to enrol or attend screening whereas worry could instead demotivate attendance among men. Higher reported anxiety among women has been observed outside of the screening context (34), as has cancer fear (35), and so it is also possible that this reflects a broader tendency or even response bias, where women more frequently report symptoms of anxiety and cancer worry.

There is also some evidence that marital status and socioeconomic position (SEP) are associated with psychological outcomes in LDCT lung cancer screening, although this is more mixed. Participants who were not married or cohabiting and had a relatively lower level of education, reported higher anxiety and fear following screening in the PLuSS study (22), and higher depression scores at baseline in the LSUT (21). In LSUT, education level was also associated with higher anxiety scores at baseline. However, these differences were not clinically significant and were not sustained at 3 months' follow-up after adjusting for baseline scores. In UKLS, those recruited from the relatively more deprived site reported poorer psychological outcomes than those living within the more affluent site, differences which persisted over time although again, the absolute differences were not clinically relevant (20). No studies have yet reported differences by marital status and education in the longer term. Furthermore, and like female gender, low education level has been associated with higher selfreported cancer fear in the general population (35), and both education and marital status with depression $(36,37)$. Further research is needed to understand the origins of these observed gender, SEP and marital status differences to ensure the appropriate support is provided to mitigate any potential increased propensity to experience screeninginduced distress. It will also be important to investigate whether these factors differentiate psychological responses to the type of LDCT result received as to date, research has focussed on differences among the screening cohort overall.

\section{Smoking status}

There are mixed findings concerning the impact of smoking status on screening-induced distress. The UKLS, PLuSS and the Stony Brook Cancer Center studies found current smoking status to be associated with higher worry, fear and distress (respectively) about lung cancer relative to former smokers following screening $(20,22,29)$. However, smoking status did not differentiate any of the psychological outcomes within the LSUT sample (21) nor anxiety within the NELSON and UKLS trials $(14,20)$. In LSUT, the eligibility criteria included very recently quit former smokers ( $<7$ years) which may have made current and former smokers more comparable groups in terms of their emotional response to lung cancer risk. Furthermore, the lack of association with generic measures of anxiety in NELSON and UKLS suggests that the impact of smoking status is limited to lung cancer-specific distress. Indeed, with regards to HRQoL, both current smoking status and number of pack-years were predictive of poorer scores on physical dimensions in the NELSON trial (13), but there were no differences for the mental health dimension. The reasons for current smokers' higher self-reported distress about lung cancer following screening are unknown. It is likely that current smokers experience negative outcomes for lung cancer among their family and social networks relatively more frequently than former smokers (38) and worry more often about their risk of the disease. In support, research has shown that current smokers are more likely to endorse fatalistic and negative beliefs about lung cancer (39-41) and report worrying about lung cancer often (40).

\section{Health beliefs}

There is some evidence that pre-existing beliefs about lung cancer and perceptions of personal risk, help to explain individual differences in psychological responses to screening. A qualitative study of individuals who took part in a real-world demonstration pilot of LDCT lung cancer screening as part of LSUT found that the ways in which individuals responded to screening appeared to depend on their perceived risk and health status, perceived 
stigmatisation of smoking-related risk, and fatalistic perceptions of lung cancer as a disease (9). Indeed, in the NELSON trial, those with high affective risk perceptions (how one feels about their risk of lung cancer) experienced intrusive thoughts more frequently that those with a low affective risk perception; both the day before screening and 6 months after screening (42). Those with pre-existing lung disease in the Stony Brook Cancer Center study, who may hold more negative perceptions of their health status, also had increased odds of experiencing lung cancer-specific distress following screening (29). The potential role of these beliefs is particularly important for future research to understand because if they are instrumental in explaining sociodemographic and smoking-related differences in psychological response, they could provide modifiable targets for interventions designed to promote positive psychological outcomes for screening.

\section{Intervening to promote psychological well-being}

The ways in which individuals respond to screening are potentially modifiable via service design and delivery. It is therefore important to understand any potential positive or negative psychological effects of LDCT lung cancer screening, their aetiology, and the characteristics of potentially vulnerable groups, so that any adverse responses can be minimised and adaptive responses maximised, preemptively. This might include tailored strategies designed for those who may benefit from supportive interventions, including women, those of a lower SEP, current smokers and those with abnormal results.

\section{Person-centred communication throughout the screening pathway: invitation and eligibility}

While there have been no interventional or observational studies of effective strategies for promoting psychological well-being in the lung cancer screening setting, there are qualitative data implicating quality, person-centred communication throughout the screening pathway. During interviews with individuals who had undergone LDCT screening, communication appeared to be important from the very outset, including during invitation and risk-based eligibility assessment (9). Indeed, given the evidence for potentially adverse psychological responses among those deemed ineligible for screening, it may also be important to develop strategies for communicating ineligibility at a population as well as individual level. For example, information about alternative risk management strategies such as smoking cessation and lung symptom awareness should be made available when ineligibility is disclosed to an individual seeking screening, to provide a means of reducing concern about lung cancer without over-reassuring that they are at 'no risk'.

\section{Person-centred communication throughout the screening pathway: results and follow-up}

Qualitative data also suggest that feeling informed about, and psychologically prepared for, each of the possible types of LDCT screening result and likelihood of needing surveillance before screening, influence how participants respond to the results they subsequently receive (9). Indeed, in UKLS, participants with a normal screen who had expected to receive an abnormal result were significantly more concerned about their result and perceived the test to be less accurate than those who had expected a normal result (43). Those receiving an abnormal result were most concerned regardless of their prior expectations. Together these findings point to the importance of preparing individuals for the different types of result, including both normal and abnormal findings, and for managing expectations for how frequently they occur and the possibility of surveillance.

\section{Person-centred communication in the context of incidental pulmonary nodules}

Useful insight for promoting well-being among individuals with abnormal LDCT findings specifically, may also be drawn from the incidental pulmonary field. A programme of research carried out by Slatore and Wiener (44) also points firmly to the role of evidence-based and personcentred communication in achieving this. Among a cohort of veterans, physician communication styles which emphasised the patient as a person (e.g., expressing interest and a positive partnership approach) significantly reduced self-reported distress (45). Participants reporting a generally higher quality of physician communication about their nodule also had statistically significantly lower distress at baseline and longitudinally (2 years' follow-up) $(45,46)$, as well as improved adherence to recommended follow-up (47). Interestingly, Slatore and colleagues (44) found some physicians avoided mentioning the possibility of cancer as a strategy intended to minimise distress, yet their data showed cancer information was more frequently perceived 
as reassuring than distressing (48). Some physicians also believed a certain amount of distress was necessary for motivating individuals to attend scheduled nodule followup appointments (49). However, distress appeared to consistently undermine patients' adherence to surveillance, with no evidence for there being any 'optimal' level of distress which motivated adherence (44). This is consistent with findings from other cancer screening settings, such as mammography and colorectal screening, where distress has been associated with poorer subsequent adherence (50).

\section{Summary}

Regardless of the type of measure used, studies to-date present no evidence of any adverse psychological impact of the screening process overall. However, those receiving indeterminate and suspicious LDCT findings do report clinically significant increases in anxiety and lung cancerspecific distress in the short-term, which appear to be resolved at long-term follow. Other characteristics for which there was some evidence of statistically raised distress included being female, of a lower SEP, not married or cohabiting, and currently smoking, as well as being ineligible for screening due to lower risk of lung cancer or denied screening following allocation to the control arm of trials. There was also early evidence that pre-existing health perceptions such as perceived risk of lung cancer, fatalism, and stigmatisation of smoking-related risk, could help to explain these individual differences. These may be important modifiable factors when intervening to minimise distress, particularly among potentially vulnerable subgroups. Indeed, research suggests that evidence-based and person-centred communication throughout the entire screening pathway, from invitation to results, should be developed and used pre-emptively to promote psychological well-being and preparedness for the different types of LDCT result. However, there was also evidence of possible psychological benefit from LDCT lung cancer screening and positive emotional and cognitive responses which deserve further study so that these can be optimised and capitalised upon.

Future research should also seek to examine psychological outcomes longitudinally within real-world LDCT lung cancer screening services for individuals taking part in repeated screening intervals, and compared, if possible, against a matched community sample. These studies should prioritise the use of situation-specific measures which are designed to be sensitive to lung cancer- specific distress (e.g., cancer worry scale), the screening and results communication 'events' (such as the IES and the COS-LC), as well as those sensitive to clinically significant anxiety (such as the HADS). There would also be benefit in quantitatively understanding the frequency, duration and magnitude of potential positive psychological responses from LDCT screening so that these can be promoted, for which psychometric development studies are needed to reliably measure these different types of response. Exploratory qualitative studies with screening participants and health care professionals are also needed to understand current communication practices, preferences and outcomes in the LDCT lung cancer screening context. This work may be especially important for those under surveillance for pulmonary nodules or undergoing diagnostic work up. This research could be directed by the conceptual model of person-centred communication for incidental pulmonary nodule proposed by Slatore and Wiener (45) in order to systematically build evidence for and test communication interventions aiming to promote psychological well-being and patient benefit.

\section{Acknowledgments}

Funding: SLQ is supported by a Cancer Research UK Population Research Fellowship (C50664/A24460).

\section{Footnote}

Provenance and Peer Review: This article was commissioned by the guest editors (Paul Van Schil and Annemiek Snoeckx) for the series "Lung cancer screening" published in Translational Lung Cancer Research. The article has undergone external peer review.

Reporting Checklist: The authors have completed the Narrative Review reporting checklist. Available at http:// dx.doi.org/10.21037/tlcr-20-1179

Peer Review File: Available at http://dx.doi.org/10.21037/ tlcr-20-1179

Conflicts of Interest: All authors have completed the ICMJE uniform disclosure form (available at http:// dx.doi.org/10.21037/tlcr-20-1179). The series "Lung cancer screening" was commissioned by the editorial office without any funding or sponsorship. SQ reports funding from Cancer Research UK and the Roy Castle 
Lung Cancer Foundation, as well as support for attending meetings from the British Thoracic Oncology Group, InHealth, and the European Respiratory Society, outside the submitted work. SJ reports funding from GRAIL Inc and Owlstone, consulting fees from Johnson and Johnson, Bard1 Lifescience and Astra-Zeneca, support for attending meetings from Astra-Zeneca and Takeda, participation on a Data Safety Monitoring Board for the Must-ARDS trial Athersys, participation on a DMEC for the REALIST trial, stock for BARD1 Lifescience, and options for Optellum, outside the submitted work. KB reports funding from the Welsh Government via Health and Care Research Wales, a personal honorarium from AstraZeneca for speaking at a workshop, and support for attending a meeting from the International Association for the Study of Lung Cancer, outside the submitted work. The authors have no other conflicts of interest to declare.

Ethical Statement: The authors are accountable for all aspects of the work in ensuring that questions related to the accuracy or integrity of any part of the work are appropriately investigated and resolved.

Open Access Statement: This is an Open Access article distributed in accordance with the Creative Commons Attribution-NonCommercial-NoDerivs 4.0 International License (CC BY-NC-ND 4.0), which permits the noncommercial replication and distribution of the article with the strict proviso that no changes or edits are made and the original work is properly cited (including links to both the formal publication through the relevant DOI and the license). See: https://creativecommons.org/licenses/by-nc-nd/4.0/.

\section{References}

1. Bray F, Ferlay J, Soerjomataram I. Global Cancer Statistics 2018: GLOBOCAN Estimates of Incidence and Mortality Worldwide for 36 Cancers in 185 Countries. CA Cancer J Clin 2018;68:394-424.

2. Aberle DR, Adams AM, Berg CD, et al. Reduced lungcancer mortality with low-dose computed tomographic screening. N Engl J Med 2011;365:395-409.

3. Moyer VA. Screening for lung cancer: U.S. Preventive Services Task Force recommendation statement. Ann Intern Med 2014;160:330-8.

4. de Koning HJ, van der Aalst CM, de Jong PA, et al. Reduced Lung-Cancer Mortality with Volume CT Screening in a Randomized Trial. N Engl J Med
2020;382:503-13.

5. Wilson JMG, Jungner G. Principles and Practice of Screening for Disease. Geneva:World Health Organisation; 1968. Available online: http://apps.who.int/ iris/bitstream/10665/37650/1/WHO_PHP_34.pdf

6. Andermann A, Blancquaert I, Beauchamp S, et al. Revisiting Wilson and Jungner in the genomic age: $\mathrm{A}$ review of screening criteria over the past 40 years. Bull World Health Organ 2008;86:317-9.

7. Slatore CG, Sullivan DR, Pappas M, et al. PatientCentered Outcomes among Lung Cancer Screening Recipients with Computed Tomography. J Thorac Oncol 2014;9:927-34.

8. Wu GX, Raz DJ, Brown L, et al. Psychological Burden Associated With Lung Cancer Screening:A Systematic Review. Clin Lung Cancer 2016;17:315-24.

9. Kummer S, Waller J, Ruparel M, et al. Mapping the spectrum of psychological and behavioural responses to low-dose CT lung cancer screening offered within a Lung Health Check. Health Expect 2020;23:433-41.

10. Harris RP, Sheridan SL, Lewis CL, et al. The harms of screening:a proposed taxonomy and application to lung cancer screening. JAMA Intern Med 2014;174:281-5.

11. Gareen IF, Duan F, Greco EM, et al. Impact of lung cancer screening results on participant health-related quality of life and state anxiety in the National Lung Screening Trial. Cancer 2014;120:3401-9.

12. van den Bergh KAM, Essink-Bot M, Bunge EM, et al. Impact of computed tomography screening for lung cancer on participants in a randomized controlled trial (NELSON trial). Cancer 2008;113:396-404.

13. van den Bergh KAM, Essink-Bot ML, Borsboom GJJM, et al. Short-term health-related quality of life consequences in a lung cancer CT screening trial (NELSON). Br J Cancer 2010;102:27-34.

14. van den Bergh KAM, Essink-Bot ML, Borsboom GJJM, et al. Long-term effects of lung cancer computed tomography screening on health-related quality of life:the NELSON trial. Eur Respir J 2011;38:154-61.

15. Taghizadeh N, Tremblay A, Cressman S, et al. Healthrelated quality of life and anxiety in the PAN-CAN lung cancer screening cohort. BMJ Open 2019;9:e024719.

16. McRonald FE, Yadegarfar G, Baldwin DR, et al. The UK Lung Screen (UKLS): demographic profile of first 88,897 approaches provides recommendations for population screening. Cancer Prev Res (Phila) 2014;7:362-71.

17. Aberle DR, Adams AM, Berg CD, et al. Baseline characteristics of participants in the randomized national 
lung screening trial. J Natl Cancer Inst 2010;102:1771-9.

18. Hestbech MS, Siersma V, Dirksen A, et al. Participation bias in a randomised trial of screening for lung cancer. Lung Cancer 2011;73:325-31.

19. Ali N, Lifford KJ, Carter B, et al. Barriers to uptake among high-risk individuals declining participation in lung cancer screening: a mixed methods analysis of the UK Lung Cancer Screening (UKLS) trial. BMJ Open 2015;5:e008254.

20. Brain K, Lifford KJ, Carter B, et al. Long-term psychosocial outcomes of low-dose CT screening: results of the UK Lung Cancer Screening randomised controlled trial. Thorax 2016;71:996-1005.

21. Kummer S, Waller J, Ruparel M, et al. Psychological outcomes of low-dose CT lung cancer screening in a realworld multi-site demonstration screening pilot:The Lung Screen Uptake Trial. Thorax 2020;75:1065-73.

22. Byrne MM, Weissfeld J, Roberts MS. Anxiety, fear of cancer, and perceived risk of cancer following lung cancer screening. Med Decis Making 2008;28:917-25.

23. Kaerlev L, Iachina M, Pedersen J, et al. CT-Screening for lung cancer does not increase the use of anxiolytic or antidepressant medication. BMC Cancer 2012;12:188.

24. Brodersen J, Thorsen H, Kreiner S. Consequences of Screening in Lung Cancer: Development and Dimensionality of a Questionnaire. Value Health 2010;13:601-12.

25. Rasmussen JF, Siersma V, Pedersen JH, et al. Psychosocial consequences in the Danish randomised controlled lung cancer screening trial (DLCST). Lung Cancer 2015;87:65-72.

26. Horowitz M, Wilner N, Alvarez W. Impact of Event Scale:a measure of subjective stress. Psychosom Med 1979;41:209-18.

27. Rasmussen JF, Siersma V, Malmqvist J. Psychosocial consequences of false positives in the Danish Lung Cancer CT Screening Trial: a nested matched cohort study. BMJ Open 2020;10:e034682.

28. Lerman C, Daly M, Sands C, et al. Mammography Adherence and Psychological Distress Among Women at Risk for Breast Cancer. JNCI J Natl Cancer Inst 1993;85:1074-80.

29. Plank A, Nemesure B. Prevalence and risk factors of elevated distress among patients undergoing screening for lung cancer. J Nurs Educ Pract 2016;6:65-71.

30. Zeliadt SB, Heffner JL, Sayre G, et al. Attitudes and Perceptions About Smoking Cessation in the Context of Lung Cancer Screening. JAMA Intern Med
2015;175:1530-7.

31. Park ER, Gareen IF, Jain A, et al. Examining whether lung screening changes risk perceptions: National Lung Screening Trial participants at 1-year follow-up. Cancer 2013;119:1306-13.

32. Park ER, Ostroff JS, Rakowski W, et al. Risk perceptions among participants undergoing lung cancer screening: baseline results from the National Lung Screening Trial. Ann Behav Med 2009;37:268-79.

33. Park ER, Streck JM, Gareen IF, et al. A qualitative study of lung cancer risk perceptions and smoking beliefs among national lung screening trial participants. Nicotine Tob Res 2014;16:166-73.

34. Hinz A, Herzberg PY, Lordick F, et al. Age and gender differences in anxiety and depression in cancer patients compared with the general population. Eur J Cancer Care (Engl) 2019;28:e13129.

35. Vrinten C, van Jaarsveld CHM, Waller J, et al. The structure and demographic correlates of cancer fear. BMC Cancer 2014;14:597.

36. Bjelland I, Krokstad S, Mykletun A, et al. Does a higher educational level protect against anxiety and depression? The HUNT study. Soc Sci Med 2008;66:1334-45.

37. Lorant V, Croux C, Weich S, et al. Depression and socioeconomic risk factors:7-year longitudinal population study. Br J Psychiatry 2007;190:293-8.

38. McCutchan G, Hiscock J, Hood K, et al. Engaging highrisk groups in early lung cancer diagnosis: A qualitative study of symptom presentation and intervention preferences among the UK's most deprived communities. BMJ Open 2019;9:e025902.

39. Quaife SL, Mcewen A, Janes SM, et al. Smoking is associated with pessimistic and avoidant beliefs about cancer: Results from the International Cancer Benchmarking Partnership. Br J Cancer 2015;112:1799-804.

40. Quaife SL, Marlow LAV, McEwen A, et al. Attitudes towards lung cancer screening within socioeconomically deprived and heavy smoking communities: informing screening communication. Health Expect 2017;20:563-73.

41. Smits SE, McCutchan GM, Hanson JA, et al. Attitudes towards lung cancer screening in a population sample. Health Expect 2018;21:1150-8.

42. Bunge EM, van den Bergh KAM, Essink-Bot ML, et al. High affective risk perception is associated with more lung cancer-specific distress in CT screening for lung cancer. Lung Cancer 2008;62:385-90.

43. Dunn CE, Edwards A, Carter B, et al. The role of screening expectations in modifying short-term 
psychological responses to low-dose computed tomography lung cancer screening among high-risk individuals. Patient Educ Couns 2017;100:1572-9.

44. Slatore CG, Wiener RS. A Small Problem for Many, Severe Distress for Some. Chest 2018;153:1004-15.

45. Slatore CG, Wiener RS, Golden SE, et al. Longitudinal assessment of distress among veterans with incidental pulmonary nodules. Ann Am Thorac Soc 2016;13:1983-91.

46. Slatore CG, Golden SE, Ganzini L, et al. Distress and patient-centered communication among veterans with incidental (not screen-detected) pulmonary nodules: A cohort study. Ann Am Thorac Soc 2015;12:184-92.

47. Moseson EM, Wiener RS, Golden SE, et al. Patient and clinician characteristics associated with adherence a cohort study of veterans with incidental pulmonary nodules. Ann
Am Thorac Soc 2016;13:651-9.

48. Freiman MR, Clark JA, Hospital M, et al. Patients' Knowledge, Beliefs, and Distress associated with Detection and Evaluation of Incidental Pulmonary Nodules for Cancer: Results from a Multi-Center Survey. J Thorac Oncol 2016;11:700-8.

49. Golden SE, Wiener RS, Sullivan D, et al. Primary Care Providers and a System Problem: A qualitative study of clinicians caring for patients with incidental pulmonary nodules. Chest 2015;148:1422-9.

50. O'Donnell S, Goldstein B, DiMatteo MR, et al. Adherence to mammography and colorectal cancer screening in women 50-80 years of age: The role of psychological distress. Womens Health Issues 2010;20:343-9.
Cite this article as: Quaife SL, Janes SM, Brain KE. The person behind the nodule: a narrative review of the psychological impact of lung cancer screening. Transl Lung Cancer Res 2021;10(5):2427-2440. doi: 10.21037/tlcr-20-1179 